Bronchialkarzinom

\section{Simpler Fragebogen entlarvt Hochrisikopatienten}

Bechtel JJ et al. Five-year outcome of lung cancer detection in patients with and without airflow obstruction in a primary care outpatient practice. J Thorac Oncol.

2009;4:1347-1351

\section{Fragestellung}

Wie kann man ohne großen Aufwand Patienten herausfischen, die ein hohes Lungenkarzinomrisiko haben?

\section{Methodik}

In Colorado wurde im Jahre 2001 in einer Hausarztpraxis ein Screening mittels eines einfachen Fragebogens erprobt. Der kurze Fragebogen war auf Risiken, Umwelt und Genetik fokusiert. Spezifische Fragen betrafen das Rauchverhalten, das Arbeitsumfeld (Bergbau, Konstruktion oder Eisenbahn), die Chemikalienexposition und die Familienanamnese. 1256 Pati- enten (Alter über 50 Jahren) füllten den Fragebogen vollständig aus.

\section{Ergebnisse}

Bei 430 Patienten wurde das Lungenkarzinomrisiko als hoch eingeschätzt, weil sie (Ex-)Raucher und/oder beruflich belastet waren und/oder eine positive $\mathrm{Fa}$ milienanamnese hatten. Diese Patienten wurden mithilfe der Spirometrie untersucht. 126 hatten eine obstruktive Lungenkrankheit, bei 304 war das Spirometrieergebnis unauffällig.

Weiterführende Untersuchungsmethoden deckten in der Hochrisikogruppe mit obstruktiven Atemwegserkrankungen acht Lungenkarzinome auf. Bei den 304 Patienten mit normaler Atmung wurden zehn Karzinome nachgewiesen.

Insgesamt wurden bei 430 Patienten, die der einfache einseitige Fragebogen als Risikopatienten identifiziert hatte, 18 Lungenkarzinome $(4,2 \%)$ ermittelt.

\section{Kommentar}

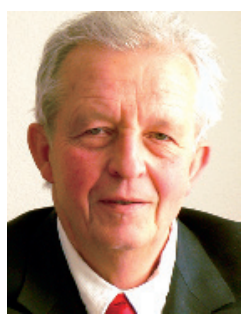

Prof. Dr. med. Kurt Malberg, Dresden-Loschwitz

Ein einfacher Fragebogen hilft, Patienten mit einem hohen Lungenkarzinomrisiko zu identifizieren. Wenn weitere Studien das bestätigen sollten, könnte diese Methode helfen, ohne großen Aufwand Patienten für das Screening auszuwählen.

\title{
Analgetika
}

\section{Erhöht Paracetamol das Asthmarisiko?}

Etminan $\mathrm{M}$ et al. Acetaminophen use and the risk of asthma in children and adults.

A systematic review and metaanalysis. Chest 2009;236:1202-1210

\section{Hintergrund und Fragestellung}

Neuere epidemiologische Studien deuten auf ein erhöhtes Asthmarisiko nach Anwendung von Paracetamol hin. Die Ergebnisse sind aber insgesamt widersprüchlich. Kanadische Forscher versuchten in einer großen Metaanalyse, die Beziehung zwischen Paracetamolanwendung und dem Asthmarisiko bei Kindern und Erwachsenen zu quantifizieren.

\section{Methodik}

Alle großen medizinischen Datenbanken wie MEDLINE (1966-2008) und EMBASE (1980-2009) wurden nach ein- schlägigen Studien durchsucht. Die Qualität der Studien wurde von zwei Gutachtern eingeschätzt und die Daten auf ein Arbeitskalkulationsblatt extrahiert. Die Metaanalyse umspannte insgesamt 19 klinische Studien - 13 Querschnitts-, vier Kohorten- und zwei FallKontroll-Studien - mit 425140 Teilnehmern.

\section{Ergebnisse}

Das Asthmarisiko war bei der Paracetamoleinnahme auf 1,63 (95-KI: 1,46-1,77) erhöht. Nahm eine Frau während der Schwangerschaft Paracetamol ein, erhöhte dies das spätere Asthmarisiko des Ungeborenen auf 1,28 (95\%-KI: 1,16-4,1).

\section{Kommentar}

Prof. Dr. med.

Kurt Malberg,

Dresden-Loschwitz

Nach diesen Resultaten erhöht Paracetamol das Asthmarisiko bei Kindern und Erwachsenen, wobei aber kein kausaler Zusammenhang bewiesen ist. Diesen Zusammenhang sollen prospektive, randomisierte, kontrollierte Studien klären. Auch die Diagnose Asthma ist klinisch zu sichern, denn oft lag nur eine Selbsteinschätzung vor.

Als wichtigste Erklärung diskutieren die Forscher eine mangelnde Hemmung der Cyclooxygenase, die ein Schlüsselferment der entzündlichen Reaktionen beim Asthma ist. Bei bereits vorhandenem Asthma sollte vor der Paracetamoleinnahme ein Arzt konsultiert werden. Für Erwachsene bietet sich als Alternative Ibuprofen an. 